\title{
miRNAs and their role in the correlation between schizophrenia and cancer (Review)
}

\author{
E. RIZOS ${ }^{1}$, N. SIAFAKAS ${ }^{2}$, E. SKOURTI ${ }^{3}$, C. PAPAGEORGIOU ${ }^{4}$, J. TSOPORIS $^{5}$, T.H. PARKER ${ }^{5}$,

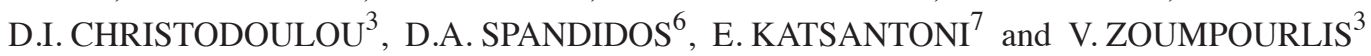

\begin{abstract}
${ }^{1}$ 2nd Department of Psychiatry, and ${ }^{2}$ Laboratory of Clinical Microbiology, National and Kapodistrian University of Athens, School of Medicine, University General Hospital 'ATTIKON', Athens $12462 ;{ }^{3}$ Unit of Biomedical Applications, Institute of Biology, Medicinal Chemistry and Biotechnology, National Hellenic Research Foundation, Athens 116 35;

${ }^{4} 1$ st Department of Psychiatry, National and Kapodistrian University of Athens, School of Medicine, 'Eginition' Hospital, Athens 115 28, Greece; ${ }^{5}$ Keenan Research Centre, Li Ka Shing Knowledge Centre, Institute of Biomedical Science,

St. Michael's Hospital, Toronto, ON M5B 1W8, Canada; ${ }^{6}$ Laboratory of Clinical Virology, Medical School, University of Crete, Heraklion 71003; ${ }^{7}$ Biomedical Research Foundation, Academy of Athens,
\end{abstract}

Hematology-Oncology Division, Athens 115 27, Greece

Received June 24, 2016; Accepted September 30, 2016

DOI: $10.3892 / \mathrm{mmr} .2016 .5853$

\begin{abstract}
Schizophrenia (SZ) and cancer (Ca) have a broad spectrum of clinical phenotypes and a complex biological background, implicating a large number of genetic and epigenetic factors. SZ is a chronic neurodevelopmental disorder signified by an increase in the expression of apoptotic molecular signals, whereas $\mathrm{Ca}$ is conversely characterized by an increase in appropriate molecular signaling that stimulates uncontrolled cell proliferation. The rather low risk of developing $\mathrm{Ca}$ in patients suffering from SZ is a hypothesis that is still under debate. Recent evidence has indicated that microRNAs (miRNAs or miRs), a large group of small non-coding oligonoucleotides, may play a significant role in the development of $\mathrm{Ca}$ and major psychiatric disorders, such as SZ, bipolar disorder, autism spectrum disorders, suicidality and depression, through their interference with the expression of multiple genes. For instance, the possible role of let-7, miR-98 and miR-183 as biomarkers for $\mathrm{Ca}$ and SZ was investigated in our previous research studies. Therefore, further investigations on the expression profiles of these regulatory, small RNA molecules and the molecular pathways through which they exert their control may provide a plausible explanation as to whether there is a correlation between psychiatric disorders and low risk of developing $\mathrm{Ca}$.
\end{abstract}

Correspondence to: Dr Emmanouil Rizos, 2nd Department of Psychiatry, National and Kapodistrian University of Athens, School of Medicine, University General Hospital 'ATTIKON', 1 Rimini Street, Athens 124 62, Greece

E-mail: erizos@med.uoa.gr

Key words: microRNA, schizophrenia, cancer

\section{Contents}

1. Introduction

2. miRNAs implicated in cancer

3. miRNAs and schizophrenia

4. miRNAs implicated in both cancer and schizophrenia

5. Conclusion

\section{Introduction}

MicroRNAs (miRNAs or miRs) comprise a class of short RNA molecules, 20-23 nucleotides in length, which play a crucial role in the regulation of gene expression at the post-transcriptional level. The majority of miRNA genes are located in intergenic regions, behaving in this case as independent transcriptional units (1). However, they may also be found in introns of coding and non-coding regions, as well as in exons of certain non-protein-coding transcripts, where their transcription is regulated together with their host exons (2).

miRNAs specifically function by targeting complementary sequences within mRNA molecules through complementary base-pairing, resulting in mRNA destabilization and degradation, or the downregulation of mRNA translation into proteins by ribosomes $(3,4)$. They exert their post-translational control on many basic cellular functions, such as growth, migration and death $(3,5,6)$. They target approximately $30 \%$ of the human genome and $70 \%$ of miRNAs identified are expressed in the central nervous system (CNS), where specific subtypes are brain or brain-region specific (7). Therefore, they play a pivotal role in neural development, differentiation and maturation, brain structure and synapse plasticity, dand their dysregulation is possibly linked to schizophrenia (SZ) by affecting cellular pathways implicated in the expression of associated genes (8-15).

Many of these genes have been linked with either an increase in the cellular apoptotic rate, or the deceleration of growth (16). 
Since SZ is a disorder characterized by an apoptosis-promoting and proliferation-inhibiting genetic activity, and cancer $(\mathrm{Ca})$ is conversely the result of uncontrolled cellular proliferation, it can be hypothesized that patients with SZ may have a lower a lower risk of developing Ca (16-19). Indeed, such an inverse correlation has been reported as early as 1909 and has been further supported more recently, including a number of population-based incidence studies (20-29). miRNAs emerge as significant etiopathogenetic factors and possible biomarkers for both disorders and may mediate this possible negative correlation, probably through their pleiotropic and epistatic function.

\section{2. miRNAs implicated in cancer}

The involvement of miRNAs in Ca has been extensively studied during the past few years, with particular focus on the potential role of these short RNA molecules in therapy and as biological markers in diagnosis and prognosis.

For instance, Zhu et al (30) demonstrated that, in prostate $\mathrm{Ca}, \mathrm{miR}-152$ suppresses the migration and invasion of Ca cells by targeting transforming growth factor- $\beta$ (TGF- $\beta$ ). Moreover, Qu et al (31) found that both miR-182 and miR-203 induce mesenchymal-to-epithelial transition (MET) by targeting snail family zinc finger 2 (SNAI2) in prostate $\mathrm{Ca}$. Of note, this induction of MET is accompanied by the self-sufficiency of growth signals and the increased survival of prostate Ca cells. In addition to the above, the upregulation of miR-143 has been shown to promote prostate Ca cell migration and invasion both in vitro and in vivo (metastasis) by suppressing fibronectin type III domain containing 3B (FNDC3B). This gene is a fibronectin family member which regulates cell motility and is downregulated in tumor cells with high metastatic potential, a fact indicating its oncosuppressive role (32).

As regards colorectal $\mathrm{Ca}, \mathrm{miR}-362-3 \mathrm{p}$ has been found to induce cell cycle arrest, and a high expression of this miRNA has been associated with a good prognosis (33). Another study demonstrated that miR-29c may play a significant role as a new circulating biomarker for the prediction of the early relapse of colorectal $\mathrm{Ca}$. In that study, miR-29c expression levels were significantly higher in the samples of the non-early relapsed patients compared to the early relapsed patients (34). Furthermore, miR-218 has been shown to inhibit cell cycle progression and promote apoptosis in colorectal $\mathrm{Ca}$ through the downregulation of BMI1 polycomb ring finger (BMI1), a well-known oncogene (35). More specifically, miR-218 was demonstrated to induce cell cycle arrest in the G2 phase by suppressing CDK4 and upregulating p53, two downstream targets of BMI1 (35).

In breast $\mathrm{Ca}$, miR-30a has been shown to suppress tumor growth and metastatic potential by targeting metadherin, a molecule with a crucial role in the metastatic cascade. Particularly, miR-30a targets metadherin, leading to the inhibition of cell migration and invasion, which means that it possibly acts as an oncosuppressor molecule (36). Additionally, miR-133a has been shown to regulate the cell cycle and proliferation rate by targeting epidermal growth factor receptor (EGFR) through the Akt signal transduction pathway. Thus, miR-133a suppresses EGFR expression, leading to a reduced signal transduction through Akt molecular pathway and as a result, reduced cell cycle progression and proliferation is observed (37). Moreover,
miR-124 negatively regulates CD151, a molecule that is highly expressed in breast $\mathrm{Ca}$ cells and promotes metastasis, and is therefore characterized as a tumor suppressor molecule (38). Hwang et al (39) highlighted the promotion of epithelial-tomesenchymal transition (EMT) of breast malignant tumors via the suppression of its target, adiponectin receptor 1 . Furthermore, miR-26a has been shown to inhibit cell proliferation and migration by suppressing the expression of myeloid cell leukemia 1 (MCL-1), an anti-apoptotic molecule, whereas miR-153 induces apoptosis in the aggressive breast Ca cell line, MDA-MB-231 (40). miR-506 seems to regulate TGF- $\beta$-induced EMT (41).

As regards miRNAs that target zeb expression, it has been shown that both the miR-200 family and miR-205 cooperatively regulate the expression of the E-cadherin transcriptional repressors, zebl and zeb2 (sipl). Both these molecules have been shown to play a role in the promotion of EMT. Additionally, the ectopic expression of these miRNAs leads to the opposite process of EMT, mesenchymal-epithelial transition (MET) and their expression is often lost in invasive breast $\mathrm{Ca}$ cell lines with the mesenchymal phenotype (42).

Moreover, it has been found that the enforced expression of miR-200 abrogates the capacity of metastatic lung adenocarcinoma cell lines to undergo EMT, invade and metastasize (43).

In another study (44), miR-155 was shown to facilitate TGF- $\beta$-induced EMT and tight junction dissolution, as well as cell migration and invasion. This means that miR-155 is another miRNA molecule with a significant potential to enhance EMT and, subsequently, metastasis. High expression levels of miR-155 also seem to positively correlate with invasive breast carcinomas.

Furthermore, miR-29a suppresses tristetraprolin, a regulator of epithelial polarity and metastasis. Thus, miR-29a is likely to possess an oncogenic and metastasis-promoting role in breast $\mathrm{Ca}(45)$.

A recent study (46) revealed the role of miR-132 in the migration and invasion of lung Ca cells. More precisely, this was shown to occur due to the targeting of zeb2 by miR-132.

As regards the role of miRNAs in tumors of the neural system, the expression of miR-21 has been shown to be increased, while the levels of miR-124 and miR-128 have been repeatedly shown to be decreased in glioblastoma (7).

\section{3. miRNAs and schizophrenia}

The architecture of the brain nervous system and the ability of the neurons for post-synaptic remodeling requires the implication of complex intracellular networks consisting of molecular signal tranduction systems, whereas miRNAs play an important and crucial role through their regulatory function on specific genes, therefore shaping the topography of the brain neural networks. Almost $70 \%$ of miRNAs identified have been shown to be highly or exclusively expressed in the brain, a number of which have been implicated in important aspects of neuronal function (47). Specifically, miR-124 and miR-9 have been shown to play a crucial role in neurogenesis; the overexpression of these miRNAs decreases the number of astrocytes, whereas the inhibition of these miRNAs reduces the number of neurons (48). Other genetic studies support the hypothesis that miRNAs play an important role not only 
in human brain development, but also in brain-diseases (8). Specifically, miRNAs may serve as a unifying link among the structural developmental anomalies, neurotransmitter alterations and finally, the treatment of SZ (49). In another study, the researchers compared the expression of 264 miRNAs from the pre-frontal cortex of schizophrenic patients and 21 individuals as a control non-schizophrenic sample population. The authors identified that the expression of 15 miRNAs decreased and the expression of one miRNA increased in the pre-frontal cortex of the schizophrenic patients, when compared with the control group of individuals (50). In a study on the Chinese population, even though the samples were obtained from peripheral blood, the authors found a potentially functional variant that affected pre-miR-30e and was closely associated with SZ (51).

$\mathrm{SZ}$ is a neurodevelopmental disorder and evidence of progressive clinical deterioration and neurostructural and molecular alterations following the onset of psychosis has led to the hypothesis that apoptosis may contribute to its pathophysiological background (52). Neuroimaging data from schizophrenic patients suggest progressive loss of cortical brain tissue in first-episode psychosis and the role of apoptosis on this phenomenon appears plausible (53). In addition, the data seem to indicate an increase in apoptosis in several brain cortical regions in SZ, particularly during the earlier stages of the disease $(18,54)$ and miRNAs, as part of the epigenetic mechanisms, play a crucial role in this process through their regulatoy effects on many mechanisms in the CNS networks (55).

\section{4. miRNAs implicated in both cancer and schizophrenia}

The rather rare co-morbidity of $\mathrm{Ca}$ and $\mathrm{SZ}$ is an old hypothesis which requires further investigation of miRNAs as molecules that regulate apoptotic signaling in both disorders. It is generally known that in neurodegenerative disorders, such as SZ, bipolar disorder, depression, autism, Parkinson's disease and dementia, an increase in apoptosis is observed, whereas Ca cells exhibit a decrease in apoptotic levels. In the present review, we aimed to analyze data from clinical studies implicating certain miRNA expression profiles as possible molecular pathways for the poor association between $\mathrm{Ca}$ and SZ. At the clinical level, two of the studies retrieved focused on the co-morbidity of SZ and $\mathrm{Ca}$ with respect to the role of miRNAs $(53,56)$. In the first of these studies, miRNA expression patterns were compared between patients suffering from SZ and patients with SZ and a solid tumor (56). It was shown that in total of the 345 different miRNAs which were analyzed by the miRCURY LNA miRNA array system (Exiqon A/S, Vedbaek, Denmark), only miR-183 was upregulated significantly in the first group, indicating a possible protective function of this miRNA against $\mathrm{Ca}$ in SZ patients. These results suggested the possibility that the expression level of miR-183 may be directly related to the absence of a solid tumor in the presence of SZ. On the contrary, the absence of miR-183 expression in the group of patients with SZ and Ca may be an indication that this miRNA is a protective factor against $\mathrm{Ca}$. Additionally, this finding is in line with other studies which support the anti-mitotic properties of miR-183 through its involvement in the modulation of different stages of apoptosis and autophagy-related genes $(57,58)$. Specifically, the knockdown of miR-183 expression has been shown to induce autophagic cell death in medullary thyroid $\mathrm{Ca}$ through the regu- lation of certain tumor suppressor signaling pathways (59), while in another study, the overexpression of miR-183 was shown to correlate with the metastatic potential of lung Ca cells (60). Furthermore, the overexpression of miR-183 has been shown to inhibit the migration and invasion of lung Ca cells. Thus, miR-183 seems to play a tumor suppressor role, possibly by activating the expression of tumor suppressor genes that control cell differentiation or apoptosis (61). In another study, a group of patients suffering from $\mathrm{Ca}$ only participated in conjunction with two other groups: one consisting of patients with SZ only and another one of patients with SZ and $\mathrm{Ca}$ (53). A substantial downregulation of let-7p-5p, miR-98-5p and of miR-183-5p was observed in those with a solid tumor or with a solid tumor and SZ, but not in those with SZ alone, providing an indication of their role in the occurrence of $\mathrm{Ca}$. High expression levels of these miRNAs may possibly lead to high levels of apoptosis, and may thus enable the incidence of SZ. Additionally, the observed low levels of expression of the same miRNAs in a sample of patients suffering from SZ and Ca possibly caused a decrease in apoptotic activity and thus, made them vulnerable to the development of malignant tumors. Furthermore, this may indicate the development of SZ due to other molecular pathways independent of these miRNAs. Let-7 is an miRNA that has been shown to play a role in other neurodegenerative disorders, such as Parkinson's disease and Alzheimer's disease. However, this was the first clinical study to implicate this specific miRNA in SZ (53).

Finally, the possibility of an miRNA-mediated association between the two disorders has been further highlighted by Taba rés-Seisdedos and Rubenstein (62) who supported that chromosome $8 \mathrm{p}$ might be a copy-number variant $(\mathrm{CNV})$ hub for both $\mathrm{SZ}$ and $\mathrm{Ca}$. This chromosomic region has a well-known genetic significance for both tumorigenesis and neuropsychiatric disorders, including oncogenes, tumor suppressor genes and at least 7 miRNA coding sequences.

\section{Conclusion}

In this review, we attempted to investigate the intervention of miRNAs in the comorbidity of $\mathrm{Ca}$ and $\mathrm{SZ}$ on the basis of the hypothesis that the incidence of malignancies in patients with $\mathrm{SZ}$ is reduced. In doing so, one should keep in mind that $\mathrm{Ca}$ constitutes a heterogeneous group of conditions of multivalent etiopathogenesis, with different responses to environmental, immunochemical and hormonal stimuli. Several miRNAs have been found to be either upregulated or downregulated, depending on the Ca type, and the miRNA pathways in tumorigenesis have not yet been fully elucidated (61). Similarly, it has been hypothesized that SZ genetics follow a polygenic model which includes both shared genetic abnormalities and variants that may contribute to different subtypes and to the heterogeneity of the disorder (63-65). Therefore, the pattern of the SZ-associated miRNAs is complex and their exact function largely unknown (66).

In practice, this means that the utility of a single miRNA is limited in predicting the risk for Ca in patients with SZ. A group of miRNAs which constitutes the 'signature' of a disorder would provide more robust evidence when investigating the molecular basis of phenotypic correlations. Such a task is particularly challenging for SZ, since there is no 
identified specific genetic profile of the disease (12). Moreover, technicalities concerning the methods of miRNA measurement and the tissue origin explain at least partially the lack of an accordance thus far on a precise SZ-related miRNA group. In particular, microarray platforms, northern blot analysis, PCR or a combination of these have all been used in different studies. As regards the specimen source, the analyses of brain tissue including dorsolateral pre-frontal cortex (DLPFC), frontal cortex (FC), superior temporal gyrus (STG) and hippocampus, of cerebrospinal fluid and of peripheral blood mononuclear cells, including genome-wide association studies (GWAS) have been utilized. Antipsychotic treatment also has an effect on the levels of some miRNAs, so the timing of the sampling affects the result $(67,68)$.

Hence, these limitations need to be considered when looking into the miRNA-intervened correlation of the two conditions. Ideally, future research should focus on more targeted comparisons including a larger sample size. Although there is evidence that there are common biological background and mechanisms $(17,62,67,69)$, the molecular implication of miRNAs in the discussed co-morbidity remains to be further explored in order to shed light on their promising role as a diagnostic, prognostic and therapeutic target.

\section{References}

1. Lagos-Quintana M, Rauhut R, Lendeckel W and Tuschl T: Identification of novel genes coding for small expressed RNAs. Science 294: 853-858, 2001.

2. Rodriguez A, Griffiths-Jones S, Ashurst JL and Bradley A: Identification of mammalian microRNA host genes and transcription units. Genome Res 14 (10A): 1902-1910, 2004.

3. Bartel DP: MicroRNAs: Target recognition and regulatory functions. Cell 136: 215-233, 2009.

4. Fabian MR, Sonenberg N and Filipowicz W: Regulation of mRNA translation and stability by microRNAs. Annu Rev Biochem 79: 351-379, 2010.

5. Lai EC: Micro RNAs are complementary to 3' UTR sequence motifs that mediate negative post-transcriptional regulation. Nat Genet 30: 363-364, 2002.

6. Kim VN: MicroRNA biogenesis: Coordinated cropping and dicing. Nat Rev Mol Cell Biol 6: 376-385, 2005.

7. De Smaele E, Ferretti E and Gulino A: MicroRNAs as biomarkers for CNS cancer and other disorders. Brain Res 1338: 100-111, 2010.

8. Schratt GM, Tuebing F, Nigh EA, Kane CG, Sabatini ME, Kiebler M and Greenberg ME: A brain-specific microRNA regulates dendritic spine development. Nature 439: 283-289, 2006.

9. Smirnova L, Gräfe A, Seiler A, Schumacher S, Nitsch R and Wulczyn FG: Regulation of miRNA expression during neural cell specification. Eur J Neurosci 21: 1469-1477, 2005.

10. Dogini DB, Ribeiro PA, Rocha C,Pereira TC and Lopes-Cendes I: MicroRNA expression profile in murine central nervous system development. J Mol Neurosci 35: 331-337, 2008.

11. Hansen T, Olsen L, Lindow M, Jakobsen KD, Ullum H, Jonsson E, Andreassen OA, Djurovic S, Melle I, Agartz I, et al: Brain expressed microRNAs implicated in schizophrenia etiology. PLoS One 2: e873, 2007.

12. Beveridge NJ, Tooney PA, Carroll AP, Gardiner E, Bowden N, Scott RJ, Tran N, Dedova I and Cairns MJ: Dysregulation of miRNA $181 \mathrm{~b}$ in the temporal cortex in schizophrenia. Hum Mol Genet 17: 1156-1168, 2008.

13. Cammaerts S, Strazisar M, Smets B, Weckhuysen S, Nordin A, De Jonghe P, Adolfsson R, De Rijk P and Del Favero J: Schizophrenia-Associated MIR204 Regulates Noncoding RNAs and Affects Neurotransmitter and Ion Channel Gene Sets. PLoS One 10: e0144428, 2015.

14. Zhang Y, Fan M, Wang Q, He G, Fu Y, Li H and Yu S: Polymorphisms in MicroRNA Genes And Genes Involving in NMDAR Signaling and Schizophrenia: A Case-Control Study in Chinese Han Population. Sci Rep 5: 12984, 2015.
15. Gumerov V and Hegyi H: MicroRNA-derived network analysis of differentially methylated genes in schizophrenia, implicating GABA receptor B1 [GABBR1] and protein kinase B [AKT1]. Biol Direct 10: 59, 2015.

16. Preti A and Wilson DR: Schizophrenia, cancer and obstetric complications in an evolutionary perspective-an empirically based hypothesis. Psychiatry Investig 8: 77-88, 2011.

17. Catts VS, Catts SV, McGrath JJ, Féron F, McLean D, Coulson EJ and Lutze-Mann LH: Apoptosis and schizophrenia: A pilot study based on dermal fibroblast cell lines. Schizophr Res 84: 20-28, 2006.

18. Glantz LA, Gilmore JH, Lieberman JA and Jarskog LF: Apoptotic mechanisms and the synaptic pathology of schizophrenia. Schizophr Res 81: 47-63, 2006.

19. Ni X, Trakalo J, Valente J, Azevedo MH, Pato MT, Pato CN and Kennedy JL: Human p53 tumor suppressor gene (TP53) and schizophrenia: Case-control and family studies. Neurosci Lett 388: 173-178, 2005.

20. Catts VS, Catts SV, O'Toole BI and Frost AD: Cancer incidence in patients with schizophrenia and their first-degree relatives - a meta-analysis. Acta Psychiatr Scand 117: 323-336, 2008.

21. Odegaard F: Mortality in Norwegian mental hospitals from 1916 to 1933. Acta Psychiatr Scand 11: 323-356, 1936.

22. Commissioners in Lunacy for England and Wales: Annual Report. HMSO, London, 1909.

23. Mortensen PB: The incidence of cancer in schizophrenic patients. J Epidemiol Community Health 43: 43-47, 1989.

24. Lawrence D, Jablensky AV, Holman CD and Pinder TJ: Mortality in Western Australian psychiatric patients. Soc Psychiatry Psychiatr Epidemiol 35: 341-347, 2000.

25. Barak Y, Achiron A, Mandel M, Mirecki I and Aizenberg D: Reduced cancer incidence among patients with schizophrenia. Cancer 104: 2817-2821, 2005.

26. Leucht S, Burkard T, Henderson J, Maj M and Sartorius N: Physical illness and schizophrenia: A review of the literature. Acta Psychiatr Scand 116: 317-333, 2007.

27. Chou FHC, Tsai KY, Su CY and Lee CC: The incidence and relative risk factors for developing cancer among patients with schizophrenia: A nine-year follow-up study. Schizophr Res 129: 97-103, 2011.

28. Crespi B: Autism and cancer risk. Autism Res 4: 302-310, 2011.

29. Lin GM, Chen YJ, Kuo DJ, Jaiteh LE, Wu YC, Lo TS and Li YH: Cancer incidence in patients with schizophrenia or bipolar disorder: A nationwide population-based study in Taiwan, 1997-2009. Schizophr Bull 39: 407-416, 2013.

30. Zhu C, Li J, Ding Q, Cheng G, Zhou H, Tao L, Cai H, Li P, Cao Q, Ju X, et al: miR-152 controls migration and invasive potential by targeting TGF $\alpha$ in prostate cancer cell lines. Prostate 73: 1082-1089, 2013.

31. Qu Y, Li WC, Hellem MR, Rostad K, Popa M, McCormack E, Oyan AM, Kalland KH and Ke XS: MiR-182 and miR-203 induce mesenchymal to epithelial transition and self-sufficiency of growth signals via repressing SNAI2 in prostate cells. Int J Cancer 133: 544-555, 2013.

32. Fan X, Chen X, Deng W, Zhong G, Cai Q and Lin T: Up-regulated microRNA-143 in cancer stem cells differentiation promotes prostate cancer cells metastasis by modulating FNDC3B expression. BMC Cancer 13: 61, 2013.

33. Christensen LL, Tobiasen H, Holm A, Schepeler T, Ostenfeld MS, Thorsen K, Rasmussen MH, Birkenkamp-Demtroeder K, Sieber OM, Gibbs P, et al; COLOFOL steering group: MiRNA-362-3p induces cell cycle arrest through targeting of E2F1, USF2 and PTPN1 and is associated with recurrence of colorectal cancer. Int J Cancer 133: 67-78, 2013.

34. Kuo TY, Hsi E, Yang IP, Tsai PC, Wang JY and Juo SH: Computational analysis of mRNA expression profiles identifies microRNA-29a/c as predictor of colorectal cancer early recurrence. PLoS One 7: e31587, 2012.

35. He X, Dong Y, Wu CW, Zhao Z, Ng SS, Chan FK, Sung JJ and Yu J: MicroRNA-218 inhibits cell cycle progression and promotes apoptosis in colon cancer by downregulating BMI1 polycomb ring finger oncogene. Mol Med 18: 1491-1498, 2013.

36. Zhang N, Wang X, Huo Q, Sun M, Cai C, Liu Z, Hu G and Yang Q: MicroRNA-30a suppresses breast tumor growth and metastasis by targeting metadherin. Oncogene 33: 3119-3128, 2014.

37. Cui W, Zhang S, Shan C, Zhou L and Zhou Z: microRNA-133a regulates the cell cycle and proliferation of breast cancer cells by targeting epidermal growth factor receptor through the EGFR/Akt signaling pathway. FEBS J 280: 3962-3974, 2013. 
38. Han ZB, Yang Z, Chi Y, Zhang L, Wang Y, Ji Y, Wang J, Zhao $\mathrm{H}$ and Han ZC: MicroRNA-124 suppresses breast cancer cell growth and motility by targeting CD151. Cell Physiol Biochem 31: 823-832, 2013.

39. Hwang MS, Yu N, Stinson SY, Yue P, Newman RJ, Allan BB and Dornan D: miR-221/222 targets adiponectin receptor 1 to promote the epithelial-to-mesenchymal transition in breast cancer. PLoS One 8: e66502, 2013.

40. Anaya-RuizM,CebadaJ,Delgado-López G,Sánchez-VázquezML and Pérez-Santos JL: miR-153 silencing induces apoptosis in the MDA-MB-231 breast cancer cell line. Asian Pac J Cancer Prev 14: 2983-2986, 2013.

41. Arora H, Qureshi R and Park WY: miR-506 regulates epithelial mesenchymal transition in breast cancer cell lines. PLoS One 8: e64273, 2013.

42. Gregory PA, Bert AG, Paterson EL, Barry SC, Tsykin A, Farshid G, Vadas MA, Khew-Goodall Y and Goodall GJ: The miR-200 family and miR-205 regulate epithelial to mesenchymal transition by targeting ZEB1 and SIP1. Nat Cell Biol 10: 593-601, 2008.

43. Schliekelman MJ, Gibbons DL, Faca VM, Creighton CJ, Rizvi $\mathrm{ZH}$, Zhang Q, Wong $\mathrm{CH}$, Wang $\mathrm{H}$, Ungewiss $\mathrm{C}$, Ahn YH, et al: Targets of the tumor suppressor miR-200 in regulation of the epithelial-mesenchymal transition in cancer. Cancer Res 71: 7670-7682, 2011.

44. Johansson J, Berg T, Kurzejamska E, Pang MF, Tabor V, Jansson M, Roswall P, Pietras K, Sund M, Religa P, et al: MiR-155-mediated loss of C/EBP $\beta$ shifts the TGF- $\beta$ response from growth inhibition to epithelial-mesenchymal transition, invasion and metastasis in breast cancer. Oncogene 32: 5614-5624, 2013

45. Gebeshuber CA, Zatloukal K and Martinez J: miR-29a suppresses tristetraprolin, which is a regulator of epithelial polarity and metastasis. EMBO Rep 10: 400-405, 2009.

46. You J, Li Y, Fang N, Liu B, Zu L, Chang R, Li X and Zhou Q: MiR-132 suppresses the migration and invasion of lung cancer cells via targeting the EMT regulator ZEB2. PLoS One 9: e91827, 2014

47. Cao X, Yeo G, Muotri AR, Kuwabara T and Gage FH: Noncoding RNAs in the mammalian central nervous system. Annu Rev Neurosci 29: 77-103, 2006.

48. Vo N, Klein ME, Varlamova O, Keller DM, Yamamoto T, Goodman RH and Impey S: A cAMP-response element binding protein-induced microRNA regulates neuronal morphogenesis. Proc Natl Acad Sci USA 102: 16426-16431, 2005.

49. Hunsberger JG, Austin DR, Chen G and Manji HK: MicroRNAs in mental health: From biological underpinnings to potentia therapies. Neuromolecular Med 11: 173-182, 2009.

50. Perkins DO, Jeffries CD, Jarskog LF, Thomson JM, Woods K, Newman MA, Parker JS, Jin J and Hammond SM: microRNA expression in the prefrontal cortex of individuals with schizophrenia and schizoaffective disorder. Genome Biol 8: R27, 2007.

51. Xu Y, Li F, Zhang B, Zhang K, Zhang F, Huang X, Sun N, Ren Y, Sui M and Liu P: MicroRNAs and target site screening reveals a pre-microRNA-30e variant associated with schizophrenia. Schizophr Res 119: 219-227, 2010.

52. Lewis DA and Lieberman JA: Catching up on schizophrenia: Natural history and neurobiology. Neuron 28: 325-334, 2000.

53. Rizos E, Siafakas N, Katsantoni E, Skourti E, Salpeas V, Rizos I, Tsoporis JN, Kastania A, Filippopoulou A, Xiros N, et al: Let-7, mir-98 and mir-183 as biomarkers for cancer and schizophrenia [corrected]. PLoS One 10: e0123522, 2015.
54. Jarskog LF, Glantz LA, Gilmore JH and Lieberman JA: Apoptotic mechanisms in the pathophysiology of schizophrenia. Prog Neuropsychopharmacol Biol Psychiatry 29: 846-858, 2005.

55. Shorter KR and Miller BH: Epigenetic mechanisms in schizophrenia. Prog Biophys Mol Biol 118: 1-7, 2015.

56. Rizos E, Siafakas N, Koumarianou A, Katsantoni E, Filippopoulou A, Ntounas P, Touloumis Ch, Kastania A and Zoumpourlis V: miR-183 as a molecular and protective biomarker for cancer in schizophrenic subjects. Oncol Rep 28: 2200-2204, 2012.

57. Fu LL, Wen X, Bao JK and Liu B: MicroRNA-modulated autophagic signaling networks in cancer. Int J Biochem Cell Biol 44: 733-736, 2012.

58. Li ZY, Yang Y, Ming M and Liu B; Li ZY1: Mitochondrial ROS generation for regulation of autophagic pathways in cancer. Biochem Biophys Res Commun 414: 5-8, 2011.

59. Abraham D, Jackson N, Gundara JS, Zhao J, Gill AJ, Delbridge L, Robinson BG and Sidhu SB: MicroRNA profiling of sporadic and hereditary medullary thyroid cancer identifies predictors of nodal metastasis, prognosis, and potential therapeutic targets. Clin Cancer Res 17: 4772-4781, 2011.

60. Wang G, Mao W and Zheng S: MicroRNA-183 regulates Ezrin expression in lung cancer cells. FEBS Lett 582: 3663-3668, 2008.

61. Babashah S and Soleimani M: The oncogenic and tumour suppressive roles of microRNAs in cancer and apoptosis. Eur J Cancer 47: 1127-1137, 2011.

62. Tabarés-Seisdedos R and Rubenstein JL: Chromosome 8p as a potential hub for developmental neuropsychiatric disorders: Implications for schizophrenia, autism and cancer. Mol Psychiatry 14: 563-589, 2009.

63. Docherty AR, Bigdeli TB, Edwards AC, Bacanu S, Lee D, Neale MC, Wormley BK, Walsh D, O'Neill FA, Riley BP, et al Genome-wide gene pathway analysis of psychotic illness symptom dimensions based on a new schizophrenia-specific model of the OPCRIT. Schizophr Res 164: 181-186, 2015.

64. Fanous AH and Kendler KS: Genetic heterogeneity, modifier genes, and quantitative phenotypes in psychiatric illness: Searching for a framework. Mol Psychiatry 10: 6-13, 2005.

65. Ruderfer DM, Fanous AH, Ripke S, McQuillin A, Amdur RL, Gejman PV, O'Donovan MC, Andreassen OA, Djurovic S, Hultman CM, et al; Schizophrenia Working Group of Psychiatric Genomics Consortium; Bipolar Disorder Working Group of Psychiatric Genomics Consortium; Cross-Disorder Working Group of Psychiatric Genomics Consortium: Polygenic dissection of diagnosis and clinical dimensions of bipolar disorder and schizophrenia. Mol Psychiatry 19: 1017-1024, 2014.

66. Wang J, Wang Y, Yang J and Huang Y: microRNAs as novel biomarkers of schizophrenia (Review). Exp Ther Med 8: 1671-1676, 2014

67. Xu Y, Yue W, Yao Shugart Y, Li S, Cai L, Li Q, Cheng Z, Wang G, Zhou Z, Jin C, et al: Exploring Transcription Factors-microRNAs Co-regulation Networks in Schizophrenia. Schizophr Bull 42: 1037-1045, 2016

68. Shi W, Du J, Qi Y, Liang G, Wang T, Li S, Xie S, Zeshan B and Xiao Z: Aberrant expression of serum miRNAs in schizophrenia. J Psychiatr Res 46: 198-204, 2012.

69. Cui DH, Jiang KD, Jiang SD, Xu YF and Yao H: The tumor suppressor adenomatous polyposis coli gene is associated with susceptibility to schizophrenia. Mol Psychiatry 10: 669-677, 2005. 